DOI 10.15421/4220012

УДК 539.3

Б. Д. Гераскин, Ю. Я. Годес, канд. физ.-мат. наук, И. Б. Кочеткова

\title{
МОДЕЛИРОВАНИЕ УСТАНОВИВШЕГОСЯ ДВИЖЕНИЯ НАГРУЗКИ ПО ОРТОТРОПНОЙ ПЛАСТИНЕ НА ВИНКЛЕРОВСКОМ ОСНОВАНИИ
}

Рассмотрена динамическая задача об установившемся движении нагрузки по бесконечной упругой ортотропной пластине, лежащей на основании Винклера. Для определения поля прогибов использован метод интегрального преобразования Фурье. В результате анализа сходимости интеграла, выражающего прогибы, установлен диапазон скоростей движения нагрузки, при которых реализуется установившийся процесс деформирования.

Ключевые слова: ортотропная упругая пластина; упругое основание; установившееся движение нагрузки; критическая скорость; интегральное преобразование Фурье.

Введение. Решения стационарных динамических задач о движении нагрузок по поверхностям полубесконечных упругих тел теряют смысл при достижении скоростью движения нагрузки некоторого критического значения, что указывает на невозможность реализации установившегося процесса десормирования при критической и сверхкритических скоростях $[6,7]$. Определению критических скоростей для упругих многослойных оснований посвящены работы $[1,2,5,8]$. Диапазон скоростей, при которых реализуется установившийся режим движения нагрузки по изотропной пластине на упругом основании, исследован в $[3,4]$. В настоящей статье излагается алгоритм моделирования движения нагрузки по ортотропной упругой пластине, лежащей на основании Винклера.

Постановка задачи. Рассматривается бесконечная ортотропная упругая пластина толщиной $h\left(-\infty<x_{1}^{0}, x_{2}^{0}<\infty,-h / 2<x_{3}^{0}<h / 2\right)$, лежащая на основании Винклера с коэффрициентом постели $k, x_{1}^{0} x_{2}^{0} x_{3}^{0}-$ неподвижная система координат, плоскость $x_{1}^{0} x_{2}^{0}$ которой совпадает с срединной плоскостью пластины (рис. 1). На пластину действует поперечная нагрузка, область приложения которой движется поступательно с постоянной по величине и направлению скоростью $\mathrm{v}$, вектор которой образует угол $\alpha$ с осью $x_{1}^{0}$. Подвижная система координат $x_{1} x_{2} x_{3}$ неизменно связана с областью нагружения, $x_{1}=x_{1}^{0}-\mathrm{v} t \cos \alpha$, $x_{2}=x_{2}^{0}-\mathrm{v} t \sin \alpha, x_{3}=x_{3}^{0}$, ее оси перпендикулярны плоскостям симмет- 
рии упругих свойств материала пластины. Интенсивность поперечной нагрузки $q\left(x_{1}, x_{2}\right)$ с течением времени не изменяется. Рассматривается установившийся процесс деформирования пластины, когда поле прогибов в подвижной системе координат явно от времени не зависит: $\mathrm{w}=\mathrm{w}\left(x_{1}-\mathrm{v} t \cos \alpha, x_{2}-\mathrm{v} t \sin \alpha\right)=\mathrm{w}\left(x_{1}, x_{2}\right)$. Требуется определить поле прогибов и установить диапазон скоростей движения нагрузки, при которых реализуется установившийся процесс деформирования.
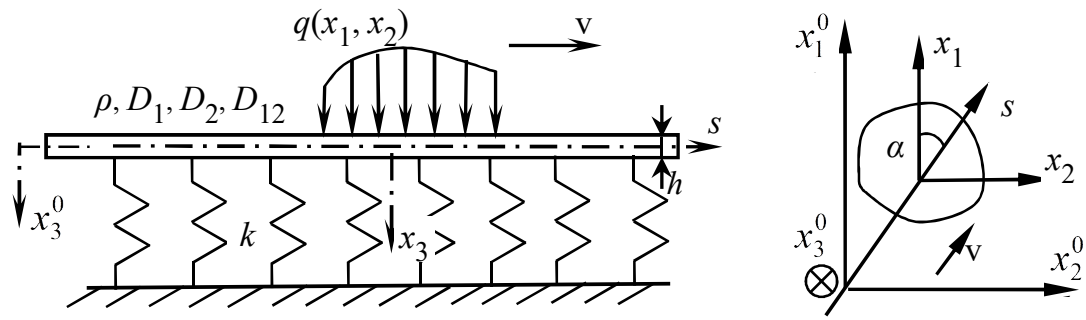

\section{Рис. 1 - Пластина на основании Винклера под действием движущейся нагрузки}

Методика решения. Так как процесс деформирования предполагается установившимся, то инерционное слагаемое в уравнении динамического изгиба ортотропной упругой пластины [10] выражается через производные прогибов по подвижным координатам, и указанное уравнение приводится к виду

$$
\begin{gathered}
D_{1} \frac{\partial^{4} \mathrm{w}}{\partial x_{1}^{4}}+2 D_{12} \frac{\partial^{4} \mathrm{w}}{\partial x_{1}^{2} \partial x_{2}^{2}}+D_{2} \frac{\partial^{4} \mathrm{w}}{\partial x_{2}^{4}}+k \mathrm{w}+ \\
+\rho h \mathrm{v}^{2}\left(\cos ^{2} \alpha \frac{\partial^{2} \mathrm{w}}{\partial x_{1}^{2}}+\sin 2 \alpha \frac{\partial^{2} \mathrm{w}}{\partial x_{1} \partial x_{2}}+\sin ^{2} \alpha \frac{\partial^{2} \mathrm{w}}{\partial x_{2}^{2}}\right)=q\left(x_{1}, x_{2}\right),
\end{gathered}
$$

где $D_{1}, D_{2}, D_{12}$ - жесткости ортотропной пластины, определяемые механическими характеристиками материала; $\rho$ - ее плотность.

Применив к уравнению (1) интегральное преобразование Фурье [9] по координатам $x_{1}, x_{2}$, получим выражение трансформанты поля прогибов

$$
D_{1} \overline{\mathrm{w}}=h^{4} \bar{q}\left(\xi_{1}, \xi_{2}\right)\left(u\left(\xi_{1}, \xi_{2}\right)\right)^{-1},
$$

где $\bar{q}\left(\xi_{1}, \xi_{2}\right)$ - трансформанта интенсивности нагрузки; фрункция $u\left(\xi_{1}, \xi_{2}\right)$ определена равенством 


$$
u\left(\xi_{1}, \xi_{2}\right)=(\xi h)^{4}\left(\cos ^{4} \varphi+\frac{m_{1}}{2} \sin ^{2} 2 \varphi+m_{2} \sin ^{4} \varphi\right)-(\xi h)^{2} \eta \cos ^{2}(\varphi-\alpha)+\kappa ;
$$

$\xi, \varphi$ - полярные радиус и угол в плоскости $\xi_{1} \xi_{2}$ параметров преобразования Фурье сответственно; $\xi_{1}=\xi \cos \varphi ; \xi_{2}=\xi \sin \varphi$. Величины

$$
\kappa=k h^{4} D_{1}^{-1}, \eta=\rho \mathrm{v}^{2} h^{3} D_{1}^{-1}, m_{1}=D_{12} D_{1}^{-1}, m_{2}=D_{2} D_{1}^{-1}
$$

представляют собой характеристики системы - безразмерные коэффициент постели, скорость и параметры ортотропии соответственно.

Поле прогибов определяется в результате применения к равенству (2) фрормулы обращения интегрального преобразования Фурье [9]:

$$
4 \pi^{2} D_{1} \mathrm{w}\left(x_{1}, x_{2}\right)=h^{4} \int_{-\infty}^{\infty} \int_{-\infty}^{\infty} \frac{\bar{q}\left(\xi_{1}, \xi_{2}\right)}{u\left(\xi_{1}, \xi_{2}\right)} e^{-i\left(\xi_{1} x_{1}+\xi_{2} x_{2}\right)} d \xi_{1} d \xi_{2} .
$$

Критическая скорость движения нагрузки. Для того чтобы формула (3) представляла решение поставленной задачи, интеграл в ее правой части должен сходиться равномерно относительно параметров $x_{1}, x_{2}$. Это требование выполняется при условии отсутствия в плоскости $\xi_{1} \xi_{2}$ нулей у функции $u\left(\xi_{1}, \xi_{2}\right)$, являющейся биквадратным трехчленом относительно $\xi$. Указанное условие приводит к неравенству

$$
\eta^{2} \cos ^{4}(\varphi-\alpha)-4 \kappa\left(\cos ^{4} \varphi+\frac{m_{1}}{2} \sin ^{2} 2 \varphi+m_{2} \sin ^{4} \varphi\right)<0,
$$

которое должно выполняться для любых значений полярного угла $\varphi$.

Таким образом, интеграл Фурье, выражающий поле прогибов, сходится равномерно, если безразмерная скорость движения нагрузки $\eta$ меньше критического значения $\eta_{*}$, определяемого равенством:

$$
\eta_{*}^{2}=4 \kappa \min _{-\pi<\varphi \leq \pi} \frac{m_{2}+2\left(m_{1}-m_{2}\right) \cos ^{2} \varphi+\left(m_{2}-2 m_{1}+1\right) \cos ^{4} \varphi}{\cos ^{4}(\varphi-\alpha)} .
$$

Реализация установившегося процесса деформирования оказывается возможной только при докритических скоростях движения нагрузки $\eta<\eta_{*}$. Значение критической скорости, задаваемое формулой (4), зависит исключительно от механических характеристик конструкции и угла между вектором скорости движения нагрузки и направлениями ортотропии.

Численная реализация решения. Для приближенного вычисления интеграла Фурье использовались адаптивные алгоритмы на основе 
квадратурных формул Гаусса и Филона, учитывающие осциллирующий характер подынтегральной фрунции. Применение таких алгоритмов позволяет избежать накопления ошибок округления и обеспечивает получение численных результатов с заданной точностью. Значение критической скорости определялось в результате численного решения задачи минимизации методом половинного деления.

В качестве иллюстрации предлагаемой методики моделирования рассмотрена задача о движении по пластине поперечной сосредоточенной силы $Q$, приложенной в начале подвижной системы координат. В этом случае трансформанта Фурье поперечной нагрузки $\bar{q}\left(\xi_{1}, \xi_{2}\right)=Q$. На рис. 2 показано изменение прогибов вдоль линии движения сосредоточенной силы при некоторых докритических значениях скорости.

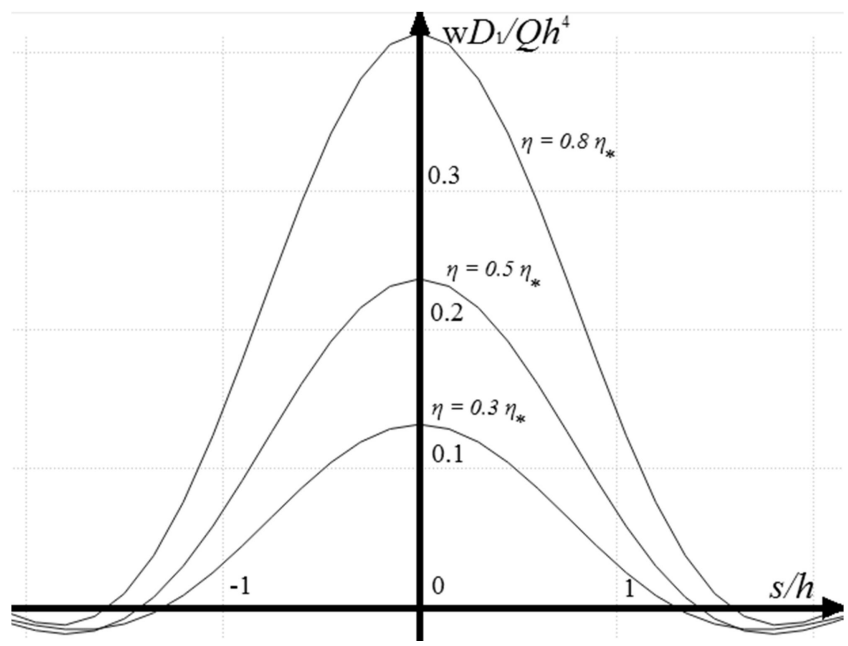

Рис. 2 - Прогибы от действия сосредоточенной силы

Параметры конструкции $\kappa=4, m_{1}=3, m_{2}=4, \alpha=30^{\circ}, \eta_{*}=5,336$, координата $s$ отсчитывается от точки приложения силы вдоль линии ее движения. Как и следовало ожидать, максимальное значение прогиба достигается в точке приложения сосредоточенной силы. С ростом скорости движения нагрузки прогибы возрастают, при стремлении скорости движения нагрузки к критической максимальное значение прогиба возрастает неограниченно.

Заключение. Построенное в настоящей работе решение стационарной динамической задачи о движении нагрузки по упругой пластине, лежащей на винклеровском основании, позволило исследовать имеющие место в таких процессах резонансные явления и установить диапазон скоростей, при которых реализуется установившийся режим деформирования. Предложена эффективная методика вычисления кри- 
тической скорости и прогибов пластины с заданной точностью, позволяющая проводить численный анализ влияния на них анизотропии материала.

Перспективным направлением дальнейших исследований является решение нестационарной задачи, когда нагрузка прикладывается к бесконечной ортотропной пластине в начальный момент времени и затем перемещается с постоянной скоростью. Значительный интерес представляет исследование условий, при которых нестационарное поле прогибов с течением времени стремится к построенному стационарному решению. Целесообразно также исследовать возможность распространения в упругой ортотропной пластине собственных волн и установить связь между их скоростями и критической скоростью установившегося движения нагрузки.

\section{БИБЛИОГРАФИЧЕСКИЕ ССЫЛКИ}

1. Вильданова С. И., Годес Ю. Я. Резонансные явления в многослойных основаниях с чередующимися однородными и неоднородными слоями // Вісн. Дніпропетр. ун-ту. Сер.: Механіка. 2008. Т. 1. Вип. 11. С. 102-116.

2. Вильданова С. И., Годес Ю. Я. Задача об установившемся движении нагрузки по неоднородному упругому основанию // Вісн. Дніпропетр. ун-ту. Сер.: Механіка. 2005. Т. 2. Вип. 9. С. $19-26$.

3. Годес Ю. Я., Кочеткова И. Б. Резонансные явления при установившемся движении нагрузки по пластине на упругом основании // Вісн. Дніпропетр. ун-ту. Сер.: Механіка. 2017. Вип. 21. С. 82-89.

4. Годес Ю. Я., Лесничий А. Н. Критическая скорость движения нагрузки по упругому основанию из пластины на полупространстве // Вопросы механики деформирования и разрушения твердых тел: сб.науч.тр. Дніпропетровськ: ДДУ, 1997. C. $22-27$.

5. Годес Ю. Я., Рябук П. В. Критическая скорость движения нагрузки по слою, сцепленному с полупространством // Вопросы механики деформирования и разрушения твердых тел: сб.науч.тр. Дніпропетровськ: ДДУ, 1998. С.19-23.

6. Гольдитейн Р. В. Волны Рэлея и резонансные явления в упругих телах // Прикладная математика и механика. 1965. Т.29. Вып. 3. С. $516-525$.

7. Гольдитейн $\boldsymbol{P}$. В. Поверхностные волны и резонансные явления в упругих телах // Соросовский образовательный журнал. 1996. № 11. С. 123 - 127.

8. Приварников А. К., Радовский Б. С. Действие подвижной нагрузки на вязкоупругое многослойное основание // Прикл. механика. 1981. Т.17. Вып. 6. С. 45-52.

9. Снеддон И. Преобразования Фурье. М.: Изд-во иностр. лит., 1955. 668 с

10. Тимошенко С. П., Войновский-Кригер С. Пластинки и оболочки. М.: Наука, 1966. $636 \mathrm{c}$.

\section{УДК 539.3}

Б. Д. Гераскін, Ю. Я. Годес, канд. фріз.-мат. наук, І. Б. Кочеткова

\section{МОДЕЛЮВАННЯ УСТАЛЕНОГО РУХУ НАВАНТАЖЕННЯ ПО ОРТОТРОПНІЙ ПЛАСТИНІ НА ВІНКЛЕРОВСЬКІЙ ОСНОВІ}

Розглянуто динамічну задачу про усталений рух навантаження по нескінченій пружній ортотропній пластині, що лежить на основі Вінклера. Для визначення поля прогинів застосовано метод інтегрального перетворення 
Фур'є. У результаті аналізу збіжності інтеграла, що виражає прогини, визначено діапазон швидкостей руху навантаження, за яких реалізується усталений процес деформування.

Ключові слова: ортотропна пружна пластина; пружна основа; усталений рух навантаження; критична швидкість; інтегральне перетворення Фур'є.

Розглядається нескінченна ортотропна пружна пластина товщиною $h$, що лежить на основі Вінклера з коефіцієнтом постелі $k$. Пластина знаходиться під дією поперечного навантаження, область прикладення якого рухається поступально з постійною швидкістю v . 3 областю навантаження незмінно зв'язана рухома система координат $x_{1} x_{2} x_{3}$, осі якої перпендикулярні площинам симетрії пружних властивостей матеріалу пластини, площина $x_{1} x_{2}$ співпадає з серединною площиною пластини, а вісь $x_{1}$ утворює кут $\alpha$ з вектором швидкості. Інтенсивність навантаження $q\left(x_{1}, x_{2}\right)$ не змінюється з часом. Розглядається усталений процес деформування, при якому поле прогинів у рухомій системі координат явно від часу не залежить.

Інерційний доданок у рівнянні динамічного згину пластини виражається через похідні прогинів по рухомих координатах. Застосовуючи інтегральне перетворення Фур'є по координатах $x_{1}, x_{2}$, знаходимо поле прогинів $\mathrm{w}\left(x_{1}, x_{2}\right)$ у вигляді інтеграла по площині параметрів перетворення $\xi_{1} \xi_{2}$. Рівномірна збіжність цього інтеграла відносно параметрів $x_{1}, x_{2}$ забезпечується відсутністю нулів у знаменника $u\left(\xi_{1}, \xi_{2}\right)$ трансформанти прогинів. Функція $u\left(\xi_{1}, \xi_{2}\right) \in$ біквадратним тричленом відносно полярного радіуса у площині параметрів перетворення, тому умова відсутності нулів призводить до нерівності $\eta<\eta_{*}$, де $\eta$ - безрозмірна швидкість руху навантаження, $\eta_{*}$ - її критичне значення. За умови $\eta<\eta_{*}$ інтеграл Фур'є, що виражає прогини, збігається рівномірно.

Таким чином, реалізація усталеного процесу деформування $є$ можливою тільки при докритичних швидкостях руху навантаження. Значення критичної швидкості залежить виключно від механічних характеристик конструкції та кута між вектором швидкісті руху навантаження та напрямами ортотропії.

Для наближеного обчислення інтеграла Фур'є застосовано адаптивні алгоритми на основі квадратурних формул Гауса і Філона, що враховують осцилюючий характер підінтегральної функції. Такі алгоритми забезпечують одержання числових результатів із заданою точністю. Значення критичної швидкості знайдено як результат числового розв'язання задачі мінімізації методом ділення навпіл. Запропоновану методику моделювання проілюстровано результатами для випадку руху по пластині зосередженої сили, що рухається з докритичними швидкостями. Продемонстровано зростання прогинів при наближенні швидкості до критичного значення. 
B. D. Heraskin, Yu. Ya. Hodes, PhD (Phys.-Math.), I. B. Kochetkova

\section{MODELLING OF STEADY-STATE LOAD MOTION ALONG AN ORTHOTROPIC PLATE ON WINKLER FOUNDATION}

Dynamic problem of a load steady-state motion is considered for an infinite elastic orthotropic plate on Winkler foundation. Fourier integral transformation is used to obtain the deflections. The Fourier integrals convergence analysis allows to find the velocities diapason in which the steady-state deformation process can be carried out.

Keywords: orthotropic elastic plate; elastic foundation; steady-state load motion; critical velocity; Fourier integral transformation.

An infinite elastic orthotropic plate on Winkler foundation is considered, $h$ is the plate thickness, $k$ is the bed coefficient. A lateral load is applied to the plate, motion of the loading area is translational with the constant velocity $\mathrm{v}$. The movable coordinate system $x_{1} x_{2} x_{3}$ is connected to the loading area, coordinate axes are perpendicular to the planes of elastic symmetry for the plate material, $x_{1} x_{2}$ is the middle plane for the plate, the velocity direction forms the angle $\alpha$ with the $x_{1}$-axis. The load intensity $q\left(x_{1}, x_{2}\right)$ does not change in time. The deformation process is considered to be steadystate: the deflection field does not depend evidently on time in the movable coordinate system.

The time derivative of the deflection in the equation of the plate dynamic bending can be expressed in terms of derivatives respect to the moving coordinates. After applying Fourier integral transformation by coordinates $x_{1}$ , $x_{2}$ we obtain the deflection field $\mathrm{w}\left(x_{1}, x_{2}\right)$ as integral over the plane of the transformation parameters $\xi_{1} \xi_{2}$ :

$$
4 \pi^{2} D_{1} \mathrm{w}\left(x_{1}, x_{2}\right)=h^{4} \int_{-\infty}^{\infty} \int_{-\infty}^{\infty} \frac{\bar{q}\left(\xi_{1}, \xi_{2}\right)}{u\left(\xi_{1}, \xi_{2}\right)} e^{-i\left(\xi_{1} x_{1}+\xi_{2} x_{2}\right)} d \xi_{1} d \xi_{2} .
$$

For this integral the uniform convergence with respect to $x_{1}, x_{2}$ is ensured if the deflections transform denominator $u\left(\xi_{1}, \xi_{2}\right)$ has no zeros. Function $u\left(\xi_{1}, \xi_{2}\right)$ is a biquadratic trinomial with respect to the polar radius in the plane of the transformation parameters, and so the condition for the zeros absence leads to inequality $\eta<\eta_{*}$, where $\eta$ is the dimensionless velocity of the load, $\eta_{*}$ is its critical value

$$
\eta_{*}^{2}=4 \kappa \min _{-\pi<\varphi \leq \pi} \frac{m_{2}+2\left(m_{1}-m_{2}\right) \cos ^{2} \varphi+\left(m_{2}-2 m_{1}+1\right) \cos ^{4} \varphi}{\cos ^{4}(\varphi-\alpha)},
$$


where $\kappa$ is the dimensionless bed coefficient; $m_{1}, m_{2}$ are the dimensionless orthotropy parameters.

Thereby the steady-state deformation process can be carried out only at subcritical velocities. The critical velocity value depends only on the mechanical characteristics of the structure and the angle between the velocity and the orthotropy directions.

The effective method is proposed to calculate deflections accurately. The adaptive algorithm based on Gauss and Filon quadratures allows to take into account oscillation of the integrand. The proposed technique is illustrated by the results for the case of concentrated force moving with the subcritical velocities. The maximum deflection increases when velocity tends to the critical value.

\section{REFERENCES}

1. Vildanova S.I., Hodes Yu.Ya. Resonance phenomena in the multilayered foundations with the alternate homogeneous and nonhomogeneous layers // Bulletin of Dnipropetr. Univ. Ser.: Mechanika. 2008. Vol. 1. Is. 11. P. 102-116. (in Russian).

2. Vildanova S.I., Hodes Yu.Ya. The problem of a load steady-state motion along inhomogeneous elastic foundation // Bulletin of Dnipropetr. Univ. Ser.: Mechanika. 2005. Vol. 2. Is. 9. P. 19-26. (in Russian).

3. Hodes Yu.Ya., Kochetkova I.B. Resonance phenomena at a load steady-state motion along a plate on elastic foundation // Bulletin of Dnipropetr. Univ. Ser.: Mechanika. 2017. Is. 21. P. 82-89. (in Russian).

4. Hodes Yu.Ya., Lesnichiy A.N. Critical velocity of the moving load along the elastic base from the plate on the half-space // Problems of solids deformation and fracture mechanics. Dnipropetrovsk: DSU. 1997. P. 22-27. (in Russian).

5. Hodes Yu.Ya., Riabuk P.V. Critical velocity of the moving load along the layer concateneted to half-space // Problems of solids deformation and fracture mechanics. Dnipropetrovsk: DSU. 1998. P. 19-23. (in Russian).

6. Goldshtein R.V. Rayleigh waves and resonance phenomena in elastic bodies // Applied Mathematics and Mechanics. 1965. Vol. 26. Is. 3. P. 516-525. (in Russian).

7. Goldshtein R.V. Surface waves and resonance phenomena in elastic bodies // Soros Educational Journal. 1996. No. 11. P. 123-127. (in Russian).

8. Privarnikov A.K., Radovsky B.S. Viscoelastic multilayered foundation under moving load // Applied Mechanics. 1981. Vol. 17. No. 6. P. 45-52. (in Russian).

9. Sneddon I. Fourier tranforms. Moscow: Foreign Literature Publ. House, 1955. 668 p. (in Russian).

10. Timoshenko S.P., Woinowsky-Krieger S. Theory of plates and shells. Moscow: Phys.-Math. Literature State Publ. House, 1966. 668 p. (in Russian).

Днепровский национальный университет имени Олеся Гончара

Днепр, Украина

Надійшла до редколеаї̈ 30.10.2020 